\title{
Rationale Synkopenabklärung
}

\author{
Work-up for Syncope
}

Autoren

Institute

\section{J. R. Ehrlich ${ }^{1}$, S. von Stuckrad-Barre ${ }^{2}$}

${ }^{1}$ Medizinische Klinik I, St. Josefs Hospital, Wiesbaden

${ }^{2}$ Neurologen Ingelheim, Ingelheim am Rhein

\author{
Schlüsselwörter \\ - Synkope \\ - Kipptischuntersuchung \\ - Implantierbarer Loop-Rekor- \\ der \\ - Schrittmacher \\ Key words \\ - syncope \\ - tilt table testing \\ - implantable loop recorder \\ - pacemaker
}

\section{Was ist wichtig?}

- Einteilung: Reflexsynkopen sowie durch orthostatische Hypotonie oder kardial bedingte Synkopen werden unterschieden und sind von nicht synkopalen Bewusstseinsverlusten abzugrenzen.

- Standardisiertes diagnostisches Vorgehen zur Synkopenabklärung umfasst Anamnese und körperliche Untersuchung. Unter Hinzunahme von Blutdruckmessung und Ruhe-EKG lässt sich in 50\% der Fälle eine Diagnose stellen.

- Risikostratifikation und weitere Diagnostik hinsichtlich des Vorliegens einer „kardialen“ Synkope bei unklaren Synkopen sollten mittels validierter Scores (z. B. OESIL-Score erfolgen). Im Rezidivfall ist oftmals ein subkutaner Loop-Rekorder indiziert.

- Kipptischuntersuchung sollte weniger und vor allem nicht zur Bestätigung einer reflektorischen Synkope eingesetzt werden. Die Anwendung sollte zur Klärung spezieller Fragestellungen (z. B. Hypotonieneigung bei begleitender Asystolie) erfolgen.

- Neurologische Untersuchung sollte unter dem Gesichtspunkt der Ausschlussdiagnostik erfolgen. Die Dauer der Bewusstseinsstörung und Reorientierung und der neurologische Status nach Ereignis sind hierbei entscheidend.

- Therapieansätze sind in der Regel nicht pharmakologisch, gelegentlich lassen sich orthostatische Synkopen mit Medikamenten bessern. Eine spezifische Therapie sollte bei kardialen Synkopen erfolgen.

- Schrittmachertherapie ist außer bei bradykarden Arrhythmien (als Ursache einer „kardialen“ Synkope) älteren Patienten (> 40 Jahre) mit asystolischen Reflexsynkopen vorbehalten.

- Fahreignung ist ein wichtiger Aspekt der ärztlichen Aufklärung von Synkopenpatienten. Während Patienten, die nur privat Auto fahren, in der Regel nicht mit Einschränkungen rechnen müssen, ist die Fahreignung bei Berufskraftfahrern gelegentlich eingeschränkt.

\section{Bibliografie}

DOI http://dx.doi.org/

$10.1055 / \mathrm{s}-0035-1545750$

Aktuel Kardiol 2015; 4: 86-91

(c) Georg Thieme Verlag KG

Stuttgart · New York .

ISSN 2193-5203

Korrespondenzadresse Prof. Joachim R. Ehrlich Medizinische Klinik I

St. Josefs Hospital

Beethovenstraße 20

65189 Wiesbaden

Tel.: 0611/177-1275

Fax: 0611/177-1276

\begin{tabular}{|c|c|}
\hline Glossar & \\
\hline $\mathrm{CT}$ & Computertomografie \\
\hline EKG & Elektrokardiogramm \\
\hline ICD & $\begin{array}{l}\text { implantierbarer Cardioverter- } \\
\text { Defibrillator }\end{array}$ \\
\hline ILR & implantierter Loop-Rekorder \\
\hline ISSUE-3 & $\begin{array}{l}\text { „International Study on Syncope } \\
\text { of Uncertain Etiology 3“ }\end{array}$ \\
\hline KTU & Kipptischuntersuchung \\
\hline MRT & Magnetresonanztomografie \\
\hline OESIL & $\begin{array}{l}\text { „Osservatorio Epidemiologico sulla } \\
\text { SIncope nel Lazio“ }\end{array}$ \\
\hline
\end{tabular}

\section{Einteilung von Synkopen}

Eine Synkope ist definiert als kurz anhaltende, spontan reversible Bewusstlosigkeit mit Tonusverlust durch eine passagere zerebrale Minderdurchblutung. Schätzungen zufolge (hohe Dunkelziffer) synkopieren ca. 10\% der Bevölkerung bis zum 80. Lebensjahr mindestens einmal. Während Reflexsynkopen vermehrt in der Jugend auftreten, stehen mit zunehmendem Alter kardiale (oft rhythmogene) oder durch orthostatische Hypotonie bedingte Synkopen im Vordergrund [1]. Die Europäischen Leitlinien haben eine alltagspraktische Klassifikation (und Nomenklatur) von Synkopen nach zugrunde liegenden Pathomechanismen vorgeschlagen. Reflexsynkopen haben zumeist eine typische Anamnese sowie eine beni- 


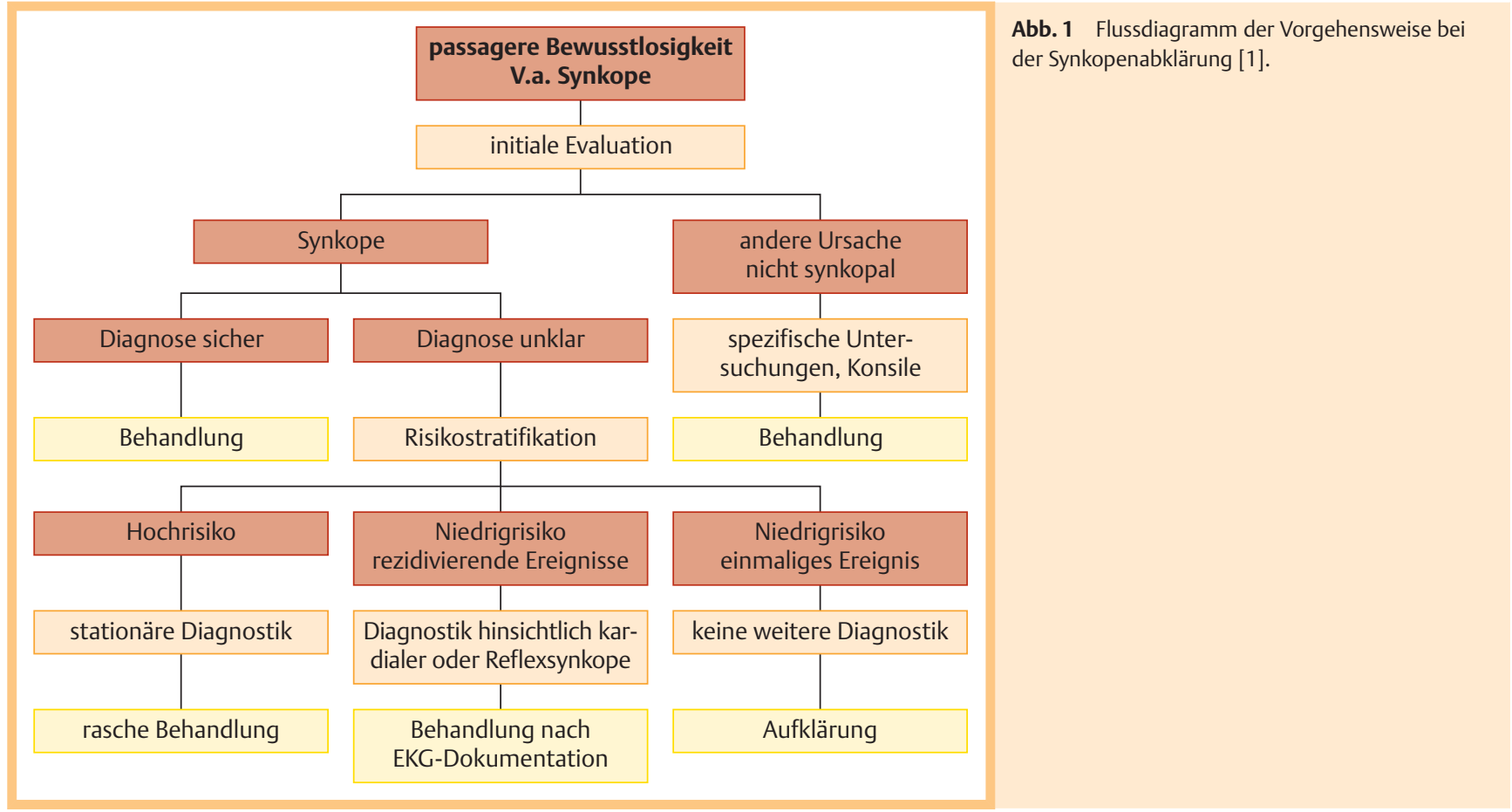

gne Prognose. Sie umfassen die als „,neurokardiogen“ oder „vasovagal“ bezeichneten Synkopen. Auch situativ bedingte Synkopen (z.B. durch Husten oder beim Anblick des eigenen Blutes) oder Synkopen durch hypersensitiven Karotissinus fallen in diese Kategorie. Hierbei kommt es durch fehlerhaftes Ansprechen des autonomen Reflexbogens zu einem verminderten Rückfluss von Blut zum Herzen (vasodepressorisch) oder zu einem verminderten Auswurf des Herzens (kardioinhibitorisch).

Als weitere Gruppe von Synkopen wurden solche durch orthostatische Hypotonie (Störung des autonomen Nervensystems, Volumenmangel, medikamentös induziert) zusammengefasst. Kardiale Synkopen (durch Arrhythmie, strukturell bedingt) deuten auf erhöhte Mortalität und ziehen zumeist stationäre Diagnostik und Behandlung nach sich.

\section{Kurzgefasst}

Synkopen werden in 3 Gruppen unterteilt:

- Reflexsynkopen umfassen u. a. „vasovagal“ bedingte Synkopen

- durch orthostatische Hypotonie bedingte Synkopen können durch Störungen des autonomen Nervensystems oder Volumenmangel entstehen

- kardiale Synkopen sind durch Arrhythmien oder strukturelle Herzerkrankungen bedingt

\section{Standardisiertes diagnostisches Vorgehen \\ $\nabla$}

Nach Klärung, ob tatsächlich eine Synkope vorlag, sollten alle Patienten einer sorgfältigen Anamnese und körperlichen Untersuchung (z.B. auskultatorisch Geräusch einer Aortenklappenstenose, geringer Hautturgor als Hinweis auf Exsikkose oder Zeichen eines Morbus Parkinson) unterzogen werden ( $\bullet$ Abb. 1 ). Die körperliche Untersuchung sollte eine Blutdruckmessung im Liegen und nach dem Aufstehen beinhalten. Darüber hinaus ist ein Ruhe-EKG anzufertigen. Das Basislabor sollte Elektrolyte, Blutzucker, Hb und CK einschließen. Das Vorliegen einer Anämie kann auf eine obere gastrointestinale Blutung hindeuten, was wiederum unerkannt mit erhöhter Mortalität einhergehen kann [1].

Die Anamnese ist die Schlüsselmaßnahme der Synkopendiagnostik. Typische Prodromi von Reflexsynkopen sind Schwindel mit aufsteigendem abdominellen Unwohlsein, das mit einem Wärmegefühl und dem Gefühl raschen Herzschlags einhergeht (autonome Aktivierung). Oftmals können Patienten, die diese Symptome kennen, sich festhalten oder noch einen Platz zum Hinsetzen aufsuchen. Auch berichten sie oftmals von Präsynkopen mit Prodromi ohne konsekutiven Bewusstseinsverlust. Ältere Patienten mit insgesamt geringerer Synkopenhäufigkeit oder kurzer oder fehlender Prodromalphase sind oftmals von kardialen Synkopen betroffen. Umgekehrt handelt es sich bei jüngeren Patienten mit typischen Prodromi und größerer Anfallshäufung oftmals eher um Reflexsynkopen [2,3].

Anamnestisch sind Synkopen ohne Prodromalsymptome oder solche, die bei körperlicher Aktivität auftreten oder von Palpitationen begleitet werden, verdächtig hinsichtlich des Vorliegens einer kardialen Synkope. Auch finden sich hier vermehrt Synkopen mit Verletzungsfolge, da keine „Vorwarnung“ des Patienten erfolgt. Synkopen durch orthostatische Hypotonie fallen u.a. durch die Tatsache auf, dass sie rasch nach einem Lagewechsel (Aufstehen) erfolgen im Gegensatz zu Reflexsynkopen, die erst nach längerem Stehen eintreten.

\section{Kurzgefasst}

Mit Anamnese, körperlicher Untersuchung, Blutdruckmessung (im Liegen und nach dem Aufstehen) und einem RuheEKG lässt sich in 50\% der Fälle eine Diagnose stellen. Synkopen, die hier initial nicht klassifiziert werden können, werden als „unklare Synkopen“ bezeichnet. 


\section{Risikostratifikation und weitere Diagnostik} $\nabla$

Sollte nach der initialen Evaluation (mit Anamnese, körperlicher Untersuchung, Blutdruckmessung, Ruhe-EKG) keine Diagnose zu stellen sein, handelt es sich um eine „unklare“ Synkope ( $\bullet$ Abb. 1). Diese Patienten sollten einer Risikostratifikation hinsichtlich Vorliegens einer kardialen Synkope und letztlich der möglicherweise erhöhten Mortalität unterzogen werden. Hierzu gibt es eine Reihe geeigneter Scores (z.B. San Francisco Syncope Rule, OESIL, EGSYS). Der OESIL-Score umfasst 4 Faktoren, die während der initialen Einordnung erfasst werden:

- EKG-Auffälligkeiten

- bekannte kardiovaskuläre Erkrankung

- Fehlen von Prodromalsymptomen

- Alter > 65 Jahre

Für Zutreffen jedes einzelnen Faktors gibt es einen Punkt; ab dem Vorliegen von 2 Faktoren handelt es sich um einen „Hochrisikopatienten“ und die weitere Diagnostik und Therapie sollte zunächst stationär durchgeführt werden [4].

Sollte keiner oder nur ein Punkt im OESIL-Score (und somit keine Hochrisikokonstellation) vorliegen, ist auch eine einzelne „unklare“ Synkope zunächst kein Grund zu weiterer Diagnostik ( Tab. 1). Im Rezidivfall sollte jedoch weiter nach Ursachen gefahndet werden. Entsprechend der aktuellen Datenlage empfiehlt sich hier „subkutane Diagnostik“ mittels implantierbarem Loop-Rekorder (ILR). Durch den Einsatz eines ILR kann eine spontane Rhythmus-Symptom-Korrelation erfolgen. Auch in Fällen, in denen bei neuerlicher Synkope keine Arrhythmien dokumentiert werden, liefert diese Diagnostik Hinweise. Registerdaten deuten auf einen hohen Prozentsatz $(\sim 78 \%)$ der Fälle, in denen ein ILR zur Diagnosefindung beitrug [5].

\section{Kurzgefasst}

Patienten mit unklaren Synkopen sollen mittels validierter Scores (z. B. OESIL-Score) hinsichtlich des Vorliegens einer kardialen Synkope und möglicher konsekutiver Mortalität risikostratifiziert werden.

\section{Stellenwert der Kipptischuntersuchung}

Die Kipptischuntersuchung (KTU) wurde 1986 zur Untersuchung ätiologisch unklarer Synkopen eingeführt [6]. Seither erfolgte weltweite Verbreitung dieses Tests in die klinische Praxis. Allerdings wuchs mit der Verbreitung auch die Erkenntnis, dass es sich um ein Diagnostikum handelt, dessen Sensitivität (78-92\%) und Spezifität (87-92\%) zwar zur Bestätigung hinsichtlich des Vorliegens einer Reflexsynkope bei suggestiver Anamnese zufriedenstellend ist, allerdings ist dies nicht der eigentlich wünschenswerte Einsatzbereich der KTU. Patienten mit klaren Reflexsynkopen benötigen in der Regel keine KTU mehr zur Diagnosestellung. Vielmehr wäre der Test wünschenswert bei Patienten mit unklarer Synkope. Hier versagt die KTU, indem sie bspw. bei fast der Hälfte der Patienten mit kardialer (rhythmogener) Synkope positiv im Sinne eines Hinweises auf eine Reflexgenese ist [7]. Die KTU produziert eine Volumenverschiebung durch die vertikale Körperposition und kann damit Personen identifizieren, die eine Empfindlichkeit für Hypotonie aufweisen. Diese Hypotonieneigung kann zu Synkopen beitragen, auch unabhängig von aufgrund einer anderen Ätiologie auftretenden Synkopen. Die KTU stellt aber in der Abgrenzung zu epileptischen Anfällen und anderen Sturzanfällen eine sinnvolle Ergänzung dar [8].
Tab. 1 Gegenüberstellung von im Allgemeinen nützlichen und in der Regel nicht nützlichen Untersuchungen zur weiteren Diagnostik unklarer Synkopen.

$\begin{array}{ll}\text { in der Regel nützliche } & \text { in der Regel nicht nützliche } \\ \text { Untersuchungen } & \text { Untersuchungen } \\ \text { Belastungs-EKG } & \text { Karotis-Doppler/Duplex } \\ \text { Karotisdruckversuch } & \text { kraniales CTund MRT } \\ \text { Echokardiogramm } & \text { Elektroenzephalogramm (EEG) } \\ \text { elektrophysiologische Untersuchung } & \text { Koronarangiografie } \\ \text { implantierbarer Loop-Recorder } & \text { Pulmonalis-Szintigrafie } \\ \text { Kipptischuntersuchung } & \text { ventrikuläre Spätpotenziale im } \\ \text { Langzeit-EKG } & \text { Signalmittelungs-EKG }\end{array}$

Eine mittels KTU diagnostizierte Hypotonieneigung kann interessanterweise unter speziellen Bedingungen für die weitere Therapie und die Prognose hinsichtlich des Ansprechens auf eine Therapie wegweisend sein. Die ISSUE-3-Studie zeigte, dass ältere Patienten mit einer im EKG dokumentierten spontanen asystolischen Reflexsynkope (kardioinhibitorische Synkope) von einer Schrittmachertherapie profitieren [9]. Allerdings war der klinische Nutzen insgesamt begrenzt. Es kam trotz der Schrittmachertherapie bei etwa 25\% der Patienten innerhalb von 2 Jahren zu einem Rezidiv. Interessanterweise traten Rezidive bei Patienten, die in der KTU einen negativen Befund aufwiesen (d.h. nicht synkopierten) nur in 5\% innerhalb der 2 Jahre auf. Daher deutet die Hypotonieneigung, die mittels KTU nachgewiesen werden kann, auf ein schlechteres Ansprechen bez. der Schrittmachertherapie. Es könnte daher erfolgversprechend sein, vor allem bei KTU-negativen Patienten mit spontaner Asystolie eine Schrittmacherindikation zu stellen [10].

\section{Kurzgefasst}

Die KTU kann bei Patienten mit Reflexsynkope in der Regel durch einen positiven Befund die Diagnose bestätigen. Allerdings ist die Untersuchung hierfür meist nicht nötig. Bei unklaren Synkopen ist die KTU nicht verlässlich wegweisend.

\section{Neurologische Untersuchung}

Wenn sich aus der Basisdiagnostik der Verdacht auf eine nicht synkopale Genese einer Bewusstseinsstörung ergibt, müssen epileptische, dissoziative Anfälle, Narkolepsie oder zerebrale Durchblutungsstörungen in die diagnostischen Überlegungen mit einbezogen werden. Hinzu kommen seltenere Funktionsstörungen (z.B. Migräne, Parasomnien). Entscheidend für die richtige Diagnose sind die sorgfältige (Fremd-)Anamnese- und klinische Befunderhebung ( Tab. 2) [11].

\section{Epileptische Anfälle}

Gezielt nachgefragt werden sollte nach Prodromi, Aura, Ausmaß der Bewusstseinsstörung, Amnesie und motorischen, sensorischen und autonomen Entäußerungen. Postiktale Veränderungen wie Lähmungen (Todd-Parese), lateraler Zungenbiss, Forellenphänomen, Muskelkater und Einnässen/-koten können weiterhelfen. Die zuverlässigsten Kriterien, einen Anfall von einer Synkope zu unterscheiden, sind die Dauer des Ereignisses und der Reorientierung. Andere Symptome wie Myoklonien werden häufig auch bei Synkopen beobachtet ( $\bullet$ Tab. 2). Weiterhin sind Provokationsfaktoren wie Schlafentzug, Alkoholexzesse oder -entzug, Drogenkonsum, Medikamentenwirkungen zu erfragen.

\section{Dissoziative (= psychogene) Anfälle}

Hilfreiche anamnestische Hinweise sind psychiatrische Vorerkrankungen und Lebensalter < 45 Jahre. Verletzungen schlie- 
Tab. 2 Differenzialdiagnose epileptischer und nicht epileptischer Anfälle.

\begin{tabular}{|c|c|c|c|}
\hline Symptome & epileptischer Anfall & dissoziativer Anfall & Synkope \\
\hline Beginn & abrupt, Aura & schleichend, variabel & langsam, Präsynkope \\
\hline Ablauf & stereotyp & sehr variabel & stereotyp \\
\hline Dauer & i. R. unter 2 Minuten & über 2 Minuten bis Stunden & unter 1 Minute \\
\hline Kloni & rhythmisch, synchron & nicht rhythmisch, asynchron & rhythmisch, synchron \\
\hline postiktale Erholung & oft langsam & variabel & schnell \\
\hline Kopfschmerzen & postiktal bei $10 \%$ & variabel & nein \\
\hline reproduzierbare Auslöser & nur Reflexepilepsien & durch Suggestion & Reize, z. B. langes Stehen, Blutabnahme \\
\hline Augen & offen (starr), Blickdeviation & geschlossen & offen, Blickdeviation \\
\hline Zungenbiss & lateral & Zungenspitze & nein \\
\hline Enuresis & häufig & möglich & selten \\
\hline Verletzungen & möglich & möglich & möglich \\
\hline Prolaktinanstieg & oft, innerhalb $2 \mathrm{~h}$ messen & möglich & möglich \\
\hline CK-Anstieg & bei Grand Mal innerhalb $24 \mathrm{~h}$ & sehr selten, bei Verletzungen & nein \\
\hline iktaler EEG-Befund & $\begin{array}{l}\text { typische Muster oder Abflachung, } \\
\text { Muskelartefakte }\end{array}$ & normal & Verlangsamung, isoelektrisch \\
\hline
\end{tabular}

ßen dissoziative Anfälle nicht aus, sondern kommen bei $>50 \%$ der Fälle vor. Anamnestisch variiert die Anfallsbeschreibung erheblich, es dominieren emotionale und situative Auslöser, und die Anfälle treten nicht aus dem Schlaf, sondern oft vor Zeugen auf. Charakteristisch ist im Vergleich zu „echten“ Synkopen, dass die betroffenen Patienten im Anfall die Augen geschlossen haben.

\section{Zerebrale Durchblutungsstörungen}

Unter den Hirninfarkten führen insbesondere die schweren Formen wie die Subarachnoidalblutung, die Basilaristhrombose oder eine Stammganglienblutung zu einer initialen Bewusstlosigkeit. Danach klart der Patient oft innerhalb von Minuten wieder auf, um sich dann sekundär zu verschlechtern. Meistens finden sich jedoch schwere fokal-neurologische Ausfälle (z.B. Meningismus, Schluckstörung, Paresen), die dann eine Einordnung und Abgrenzung zu anderen Ursachen ermöglichen. Jegliche akute Bewusstseinsstörung mit zusätzlichen fokal-neurologischen Ausfällen muss natürlich zu einer sofortigen Einweisung in eine Akutklinik mit entsprechenden diagnostischen und therapeutischen Möglichkeiten (24-h-Bildgebung, Stroke Unit usw.) führen.

\section{Narkolepsie}

Die im Rahmen der narkoleptischen Tetrade auftretenden Schlafattacken, aber auch kataplektischen Anfälle stellen ebenfalls eine Differenzialdiagnose der akuten Bewusstseinsstörung dar. Die Schlafattacken führen zu Einschlafen in häufig unpassenden Situationen. Bei der Kataplexie kommt es zu einem Tonusverlust ohne Bewusstseinsverlust. Hier finden sich anamnestisch häufig emotionale Auslöser, der Betroffene ist kontaktfähig und kennt oft seine Erkrankung, was eine Einordnung erleichtert.

\section{Seltene Ursachen, häufige Fallstricke}

$\mathrm{Zu}$ Stürzen ohne Bewusstseinsstörung können auch periphervestibuläre Funktionsstörungen führen. Im Rahmen des Morbus Menière gibt es selten $(<5 \%)$ akzentuierte Attacken, bei denen der Patient zu Boden stürzt. Auch der gutartige Lagerungsschwindel oder die Basilarismigräne können zu Gleichgewichtsstörungen mit konsekutiven Stürzen führen. Diese Erkrankungen führen alle zu keiner Bewusstseinsstörung, aber oft zu Stürzen. Andere Erkrankungen wie die Hyperventilationstetanie oder die transiente globale Amnesie gehen ebenfalls nicht mit Bewusstseinsstörungen einher [11].

\section{Kurzgefasst}

Die zuverlässigsten Kriterien, einen epileptischen Anfall von einer Synkope zu unterscheiden, sind die Dauer des Ereignisses und der Reorientierung. Zu den wichtigsten Differenzialdiagnosen aus neurologischer Sicht zählen epileptische und psychogene Anfälle.

\section{Therapieansätze}

$\nabla$

Bei Reflexsynkopen zeigen medikamentöse Therapieversuche Wirkung auf Placeboniveau. Weder für Betablocker noch für Sympathikomimetika (z.B. Midodrin) oder eine Mineralokortikoidgabe lassen sich überzeugende Wirkungen demonstrieren [1]. Allgemeinmaßnahmen - wie reichliche Flüssigkeitszufuhr, Kompressionsstrümpfe und Aktivierung der Muskelpumpe der Waden - sind wirksam.

Im Gegensatz zu Reflexsynkopen können orthostatische Synkopen mittels medikamentöser Therapie eher günstig beeinflusst werden. Hier haben Salzzufuhr und Gabe von Mineralokortikoiden einen gewissen Stellenwert. Oftmals liegt allerdings auch eine Überdosierung antihypertensiver oder diuretischer Medikation der Synkope zugrunde, deren Behandlungsansatz sich logisch ergibt.

Neben typischen bradykarden oder tachykarden Herzrhythmusstörungen, die jeweils einer spezifischen Therapie zugeführt werden müssen, bleibt bei Patienten mit rezidivierenden, unklaren Synkopen ein therapeutisches Dilemma.

Eine Schrittmachertherapie ist im Falle bradykarder Rhythmusstörungen indiziert, darüber hinaus gelegentlich älteren Patienten (> 40 Jahre) mit Reflexsynkopen und im ILR dokumentierten Asystolien zwischen 3 (symptomatisch) und 6 (asymptomatisch) Sekunden vorbehalten.

\section{Kurzgefasst}

Bei Reflexsynkopen sind medikamentöse Therapieversuche nicht erfolgversprechend. Bei orthostatischer Hypotonie kann durch Medikation eine Besserung eintreten. Schrittmachertherapie ist in der Regel kardialen rhythmogen bedingten Synkopen vorbehalten. Bei älteren Patienten und reflexbedingten asystolen Synkopen besteht ein gewisser Nutzen der Schrittmachertherapie. 


\section{Fahreignung}

$\nabla$

Eine häufig unterschätzte und dadurch leicht übersehene ärztliche Pflicht ist die Aufklärung der Patienten über eine fehlende Fahreignung mit entsprechender Dokumentation. Entscheidend für die Fahreignung sind Ätiologie und Situation, in der die Synkope auftritt, sowie die Unterscheidung zwischen Fahrern von privaten Pkw oder beruflichen Lkw-Fahrern, da letztere strengeren Regeln unterliegen. Insbesondere bei Hochrisikopatienten (z.B. kardiogene Synkopen) und Hochrisikosituationen (z. B. Gefahrguttransporter oder Gerüstarbeiten) bestehen eher Einschränkungen der Fahreignung. Hierfür sollte das sehr differenzierte Positionspapier der Deutschen Gesellschaft für Kardiologie zu Rate gezogen werden [12].

\section{Kurzgefasst}

Das Thema Fahreignung sollte in jedem Fall angesprochen und dokumentiert werden. Für die private Pkw-Nutzung besteht in aller Regel Fahreignung außer bei Hochrisikopatienten und Synkopen in Hochrisikosituationen.

\section{Fazit}

„Weniger ist mehr“: Eine gründliche Anamnese verbunden mit körperlicher Untersuchung, Blutdruckmessung und Ruhe-EKG liefert in vielen Fällen die Diagnose ohne weitere apparative Untersuchungen.

Bei rezidivierenden, ätiologisch unklaren Synkopen ist eine Risikostratifizierung notwendig, um Patienten mit hohem kardiovaskulärem und Mortalitätsrisiko zu erkennen.

Bei rezidivierenden Synkopen von Niedrigrisikopatienten sollte frühzeitig die Implantation eines subkutanen „Loop-Rekorders“ erwogen werden.

Synkopen müssen von nicht synkopalen Bewusstseinsverlusten abgegrenzt werden, da Epilepsie oder andere Erkrankungen eine spezifische Therapie erfordern.

Ein Fahrverbot ergibt sich für „Nicht-Berufsfahrer“ bei Reflexsynkopen oder Synkopen durch orthostatische Hypotonie in der Regel nicht. Kardiale Synkopen müssen in der Regel therapiert werden, um die Fahreignung wieder herzustellen.

\section{Zusammenfassung \\ $\nabla$}

Synkopen sind definiert als kurzanhaltende, spontan reversible Bewusstseinsverluste mit Tonusverlust infolge zerebraler Minderperfusion. Sie stellen häufige Probleme dar, die zur Vorstellung von Patienten in Praxen und Kliniken führen. Wenn eine Synkope entsprechend der Definition vorliegt, ist die Abgrenzung zu nicht synkopalen passageren Bewusstseinsverlusten (z.B. neurologisch, metabolisch, traumatisch) wichtig. Nach Anamnese, körperlicher Untersuchung und Ruhe-EKG kann bereits in 50\% der Fälle eine ätiologische Zuordnung erfolgen. In unklaren, rezidivierenden Fällen oder bei Patienten mit einem hohen Risiko für ein kardiovaskuläres Ereignis ist weitere Diagnostik - ggf. sogar stationär - notwendig. Der frühzeitige Einsatz eines subkutanen Loop-Rekorders erspart eine Vielzahl an unnötiger weiterer Diagnostik. Kipptischuntersuchungen können sensitiv und spezifisch eine Reflexsynkope bei Patienten mit entsprechender Anamnese bestätigen. Bei unklaren Synkopen helfen Kipptischuntersuchun- gen nicht verlässlich weiter. Stationäre Diagnostik und Therapie sollte bei Patienten erfolgen, die mittels Risikoscores (z. B. OESILScore) ein erhöhtes Risiko für ein kardiovaskuläres Ereignis oder Mortalität aufweisen. Eine neurologische Untersuchung sollte vor allem bei Fällen mit nicht synkopaler Bewusstseinstrübung erfolgen, bei denen Bewusstlosigkeit und Reorientierung länger anhalten oder ein Ereignis mit spezifischen Stigmata eines epileptischen Anfalls einhergeht (z.B. Myoklonien, Zungenbiss). Eine Schrittmachertherapie ist bei bradykardiebedingten Synkopen indiziert. Neben einer klaren Indikation bei bradykarden Rhythmusstörungen besteht eine Indikation zur Schrittmachertherapie bei Patienten mit rezidivierenden Reflexsynkopen und mittels Loop-Rekorder dokumentierten asystolen Pausen. Hier kann eine Reduktion der Rezidive erreicht werden. Ein Fahrverbot ist bei spezifischen Reflexsynkopen in der Regel nicht indiziert. Es muss jedoch unterschieden werden, ob ein Fahrer privat oder beruflich fährt. Dies spielt bei der Beurteilung der Fahreignung eine wichtige Rolle.

\section{Abstract \\ $\nabla$}

Syncope is defined as a short-lasting transient loss of consciousness with loss of motor tone due to cerebral hypoperfusion and spontaneous recovery. It must be separated from non-syncopal transient loss of consciousness (e.g. neurologically, metabolically or trauma-related). Recent guidelines categorize syncope into three groups: (a) reflex-syncope which is neurally mediated, (b) syncope due to orthostatic hypotension, (c) cardiac syncope caused by structural disease or arrhythmia. A diagnosis can usually be made in $\sim 50 \%$ of cases after taking a careful history, physical exam and registration of a resting ECG. Etiologically undefined cases should be further worked-up if they recur. Patients with unclear cause of syncope should undergo risk stratification using established scoring systems (e.g. OESIL score). In case of a high risk of cardiovascular event or death, in-hospital work-up should ensue and efforts be made to provide a timely diagnosis with subsequent therapy. Head-up tilt table testing is sensitive and specific if history suggests reflex-syncope. In unclear cases tilt-testing is not of additional diagnostic value. In recurrent cases the implantation of a subcutaneous loop-recorder should be considered early on. This tool allows for spontaneous rhythm-symptom correlation and will lead to a reduction in other non-indicated tests that would otherwise be performed. Neurological consultation is indicated in patients with complaints suggestive of non-syncopal loss of consciousness (e.g. prolonged loss of consciousness and slow re-orientation). In cases of documented cardiac arrhythmia as cause of syncope either pace-maker, ICD implant or ablation should be performed. Pacemaker therapy may be an option for elderly patients with reflex asystolic syncope and documentation of pauses by a loop recorder. Pacing can reduce number of relapses under these conditions. Driving will usually be permitted in cases of reflex-syncope; however the type of driving (private vs. professional) needs to be considered. Cardiac syncope typically needs treatment before driving can be permitted.

\section{Interessenkonflikt}

$\nabla$

Die Autoren geben an, dass kein Interessenkonflikt besteht. 


\section{Literatur}

1 Moya A, Sutton R, Ammirati $F$ et al. Guidelines for the diagnosis and management of syncope (version 2009). Eur Heart J 2009; 30: 26312671

2 Calkins H, Shyr Y, Frumin H et al. The value of the clinical history in the differentiation of syncope due to ventricular tachycardia, atrioventricular block, and neurocardiogenic syncope. Am J Med 1995; 98: 365373

3 Alboni P, Brignole M, Menozzi C et al. Diagnostic value of history in patients with syncope with or without heart disease. J Am Coll Cardiol 2001; 37: 1921-1928

4 Colivicchi F, Ammirati F, Melina D et al. Development and prospective validation of a risk stratification system for patients with syncope in the emergency department: the OESIL risk score. Eur Heart J 2003; 24: 811-819

5 Edvardsson N, Frykman V, van Mechelen $R$ et al. Use of an implantable loop recorder to increase the diagnostic yield in unexplained syncope: results from the PICTURE registry. Europace 2011; 13: 262-269

6 Kenny RA, Ingram A, Bayliss J et al. Head-up tilt: a useful test for investigating unexplained syncope. Lancet 1986; 1: 1352-1355
7 Ungar A, Sgobino P, Russo V et al. Diagnosis of neurally mediated syncope at initial evaluation and with tilt table testing compared with that revealed by prolonged ECG monitoring. An analysis from the Third International Study on Syncope of Uncertain Etiology (ISSUE-3). Heart 2013; 99: 1825-1831

8 Diehl R. Synkopen. Leitlinien der Deutschen Gesellschaft für Neurologie. AWMF-Leitlinie 2012. Im Internet: http://www.awmf.org/ leitlinien/detail/1//030-072.html; Stand: 05.03.2015

9 Brignole M, Menozzi C, Moya A et al. Pacemaker therapy in patients with neurally mediated syncope and documented asystole: Third International Study on Syncope of Uncertain Etiology (ISSUE-3): a randomized trial. Circulation 2012; 125: 2566-2571

10 Sutton R, Brignole M. Twenty-eight years of research permit reinterpretation of tilt-testing: hypotensive susceptibility rather than diagnosis. Eur Heart J 2014; 35: 2211-2212

11 von Stuckrad-Barre S, Michel R, Jost WH. Rationale Diagnostik und Differentialdiagnostik bei Synkopen. Hess Ärztebl 2010; 3: 149-156

12 Klein HH, Krämer A, Pieske BM et al. Fahreignung bei kardiovaskulären Erkrankungen. Kardiologe 2010; 4: 441-473 